14.1

\title{
Новый метод получения генераторного радионуклида Sr-82 и других медицинских радионуклидов
}

\author{
() В.Н. Пантелеев, А.Е. Барзах, Л.Х. Батист, Д.В. Федоров, В.С. Иванов, С.А. Кротов, П.Л. Молканов, \\ С.Ю. Орлов, М.Д. Селиверстов, Ю.М. Волков
}

Петербургский институт ядерной фризики им. Б.П. Константинова Национального исследовательского центра „Курчатовский институт“, 188300 Гатчина, Ленинградская обл., Россия

e-mail: panteleev_vn@pnpi.nrcki.ru

(Поступило в Редакцию 15 декабря 2017 г.)

В Петербургском институте ядерной физики Национального исследовательского центра „Курчатовский институт“ произведен запуск циклотрона (Ц-80), рассчитанного на получение протонов с энергией $40-80 \mathrm{MeV}$ и интенсивностью пучка 100-200 $\mu$ А. Основной задачей Ц-80 является получение широкого спектра медицинских радионуклидов для диагностики и терапии. Для этой цели разработан проект комплекса Радиоактивные изотопы на циклотроне Ц-80 (РИЦ-80), который будет функционировать на пучке Ц-80. Дано краткое описание комплекса РИЦ-80, а также представлены результаты использования новых методов и исследований мишенных устройств для получения генераторного радиоизотопа ${ }^{82} \mathrm{Sr}$, радиоизотопа ${ }^{67} \mathrm{Cu}$, радиоизотопов распадающихся альфа-распадом ${ }^{223} \mathrm{Ra},{ }^{224} \mathrm{Ra},{ }^{225} \mathrm{Ac}$, а также других медицинских радионуклидов. Особенностью проекта является использование в данном комплексе масс-сепаратора в режиме „в линию“ для получения ионных пучков радиоизотопов высокой чистоты, что особенно важно для медицинских приложений.

DOI: 10.21883/JTF.2018.09.46412.2601

\section{Введение}

В последние десятилетия благодаря использованию методов ядерной физики в области современной медицины возникло совершенно новое направление - ядерная медицина. Основное преимущество ядерной медицины состоит в том, что используемые в ней методы позволяют диагностировать функциональные отклонения жизнедеятельности органов на самых ранних стадиях болезни, когда человек еще не чувствует симптомы заболевания. Технологии ядерной медицины как для диагностики, так и для терапии различных заболеваний используют различные виды излучений радиоактивных нуклидов, производимых на реакторах и на ускорителях заряженных частиц. В качестве ускорителей заряженных частиц в производстве радионуклидов наиболее широко используются циклотроны, как безопасные и надежные установки. Набор циклотронных радионуклидов значительно шире и разнообразнее, чем номенклатура радионуклидов, получаемых в реакторах. Используя мишени, содержащие ${ }^{238} \mathrm{U}$, можно с достаточно высокой эффективностью получать в реакции деления протонами те же радионуклиды, что и в реакторах. При этом очень существен тот факт, что только в ускорителях заряженных частиц можно производить радионуклиды, излучающие позитроны и используемые для позитронной эмиссионной томографии (ПЭТ).

\section{1. Радиоизотопный комплекс РИЦ-80}

Ускоритель Ц-80 рассчитан на получение пучков протонов с энергией $40-80 \mathrm{MeV}$ и интенсивностью
100-200 $\mu \mathrm{A}$ [1]. Протонные пучки этого ускорителя предназначены для лечения офтальмологических заболеваний облучением злокачественных образований глаза и для получения широкого спектра медицинских радиоизотопов, используемых как для диагностики, так и для терапии различных заболеваний $[2,3]$. Протонный пучок может быть выведен на одну из трех мишенных станций, находящихся в подвальном этаже экспериментального зала циклотрона. Масс-сепаратор будет смонтирован на крайней правой по ходу протонного пучка мишенной станции и будет использоваться для производства сверхчистых пучков медицинских радионуклидов [4]. Каждая мишенная станция будет снабжена механизмом перемещения радиоактивных мишеней в специальные защищенные хранилища, а также к „горячим камерам“ для выделения необходимых нуклидов из мишенного вещества и приготовления радиофармпрепаратов. Полный список планируемых к получению радионуклидов приведен в $[5,6]$.

\section{2. Высокотемпературные методы разделения целевых радионуклидов и мишенного вещества}

Разрабатываемый метод разделения целевых радионуклидов и мишенного вещества основан на различии при определенной достаточно высокой температуре давления насыщенных паров материала мишенного вещества и атомов продуктов, образовавшихся в результате различных ядерных реакций в мишенном веществе. На рис. 1 приведены величины энергий адсорбции для ато- 


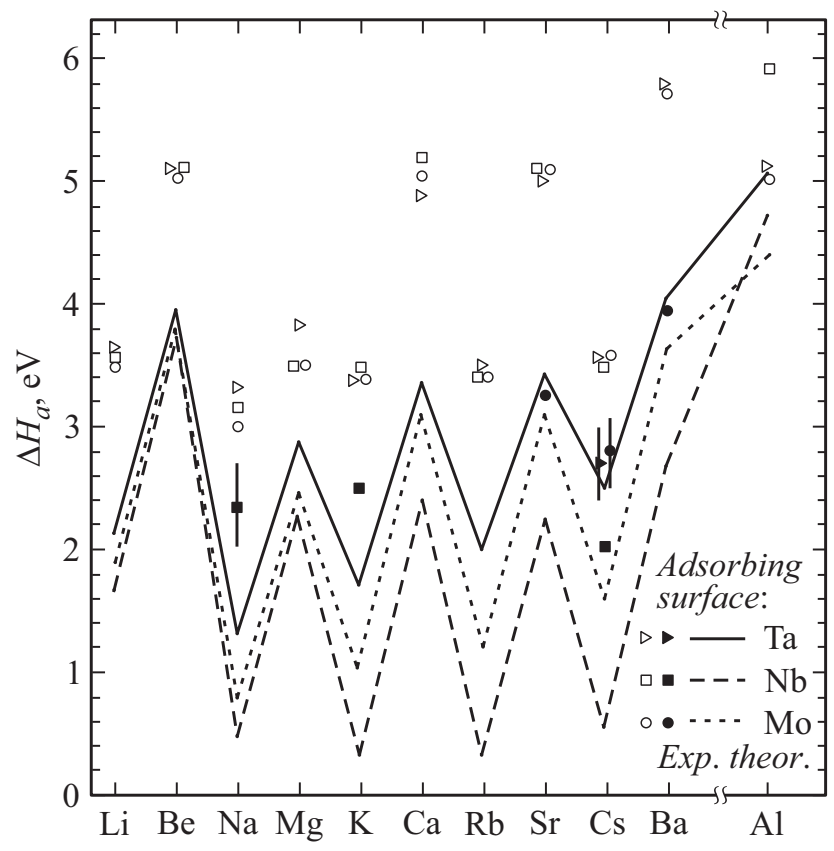

Рис. 1. Величины энергий адсорбции для атомов различных элементов на поверхностях тугоплавких металлов - молибдена, ниобия и тантала [9].

мов различных элементов на поверхностях тугоплавких металлов - молибдена, ниобия и тантала [7].

Как можно видеть из приведенного рисунка, значения величин энергии адсорбции для достаточно широкого круга элементов практически не зависят от материала адсорбирующей металлической поверхности, а зависят очень существенно, что представляется естественным, от их летучести. А так как летучесть элементов при фиксированной температуре находится в обратной корреляции с их температурами кипения (чем выше температура кипения, тем ниже летучесть элемента, чем ниже температура кипения, тем выше летучесть), то хорошим ориентиром при определении возможности разделения атомов различных элементов может служить различие в их температурах кипения, которые хорошо известны. Исходя из вышеприведенных рассуждений, для эффективного разделения мишенного вещества (металлический рубидий, температура кипения $\left.T_{b}=688^{\circ} \mathrm{C}\right)$ и радиоактивных атомов стронция (целевой радионуклид, $T_{b}=1382^{\circ} \mathrm{C}$ ) необходимо обеспечить нагрев мишенного вещества до температуры достаточно быстрого испарения (в течение нескольких часов) мишенного вещества. Так как при этой температуре радиоизотопы стронция будут вылетать значительно медленней из нагреваемого объема, то можно ожидать, что значительное количество радиоактивных атомов стронция останется в нагреваемом объеме.

При температуре около $600^{\circ} \mathrm{C}$ мишенное вещество (металлический рубидий) может быть достаточно быстро испарено. После полного испарения мишенного вещества (обычно испарение мишенного вещества с минимальными его потерями производится в балластный объем), оставшийся в мишенном объеме стронций может быть вымыт раствором кислоты или испарен на охлаждаемый коллектор при значительно более высокой температуре.

Другой, еще более универсальный метод высокотемпературного вакуумного выделения - это когда практически нет испарения мишенного вещества при температуре достаточно быстрого испарения целевого радионуклида. Достаточно быстрое испарение - это испарение с минимальными потерями, когда время выделения целевого радионуклида значительно меньше его периода полураспада. Данный метод широко используется для выделения радиоизотопов в мишенных устройствах масс-сепараторных установок, работающих в режиме „в линию“ на пучках различных бомбардирующих мишень частиц.

\section{3. Выделение ${ }^{82} \mathrm{Sr}$ из мишеней в виде порошка соли $\mathrm{RbCl}$ и металлического рубидия}

В экспериментах по получению ${ }^{82} \mathrm{Sr}$ в качестве мишенного материала использовался порошок соли хлористого рубидия, а также металлический рубидий. Радионуклид ${ }^{82} \mathrm{Sr}$ с периодом полураспада $T_{1 / 2}=25.55 \mathrm{~d}$ является генератором его дочернего изотопа ${ }^{82} \mathrm{Rb}$ $\left(T_{1 / 2}=1.25 \mathrm{~min}\right)$, который широко используется в ПЭТ диагностике. Для разделения мишенного материала и образованных в нем в качестве продуктов реакций изотопов стронция использовался новый метод „сухого“, высокотемпературного выделения $[8,9]$. После облучения в пучке протонов с энергией $1000 \mathrm{MeV}$ синхроциклотрона ПИЯФ мишенный материал помещался в стальную или ниобиевую капсулу, которая, в свою очередь, помещалась в танталово-вольфрамовый нагреватель, нагревае-

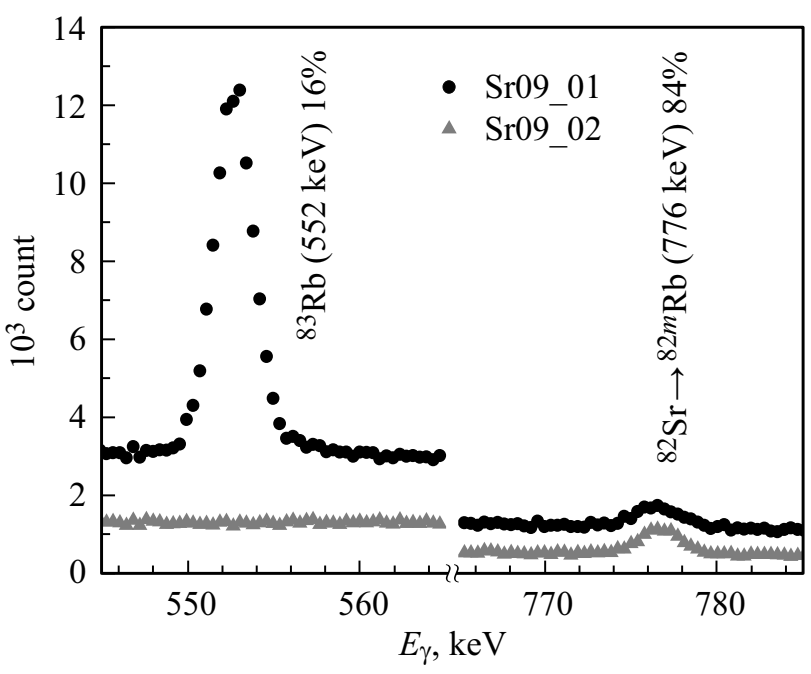

Рис. 2. Часть спектра капсулы с облученным порошком хлористого рубидия до нагрева при температуре $900^{\circ} \mathrm{C}$ (верхний спектр) и после (нижний спектр). 


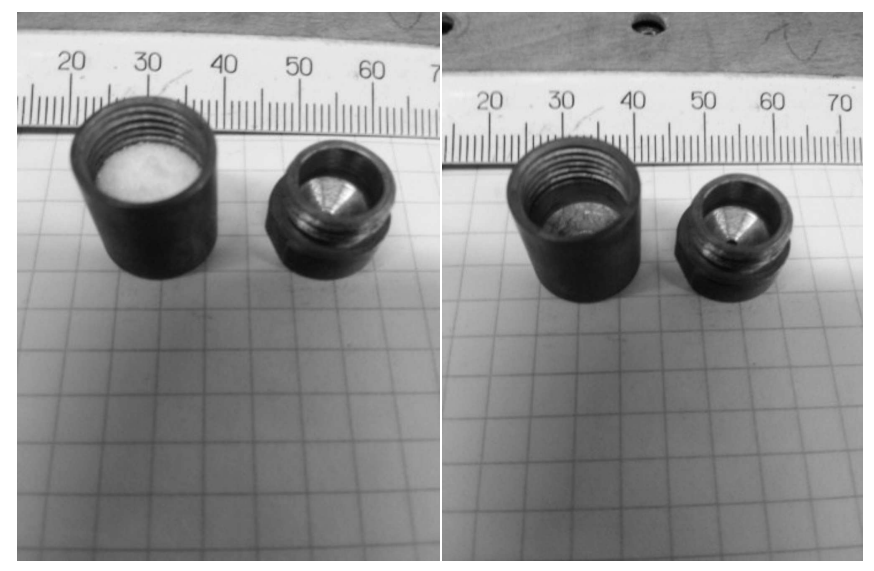

Рис. 3. Капсула с мишенным веществом до нагрева (слева), а также после нагрева, когда мишенное вещество полностью испарилось за $1 \mathrm{~h}$, что также видно на рис. 2 по полному исчезновению линии $552 \mathrm{keV}$.

мый методом резистивного нагрева, где в вакууме проводился нагрев мишенного вещества. Процесс нагрева проводился в высоком вакууме плавным повышением температуры до $900^{\circ} \mathrm{C}$ с тем, чтобы избежать неконтролируемого быстрого выхода мишенного вешества. Испарение проводилось в специальный балластный объем, позволяющий минимизировать потери мишенного вещества при его выходе из отверстия мишенной капсулы. При этой температуре процесс полного испарения мишенного вещества массой около $1 \mathrm{~g}$ занимал около $1 \mathrm{~h}$. Для контроля процесса испарения мишенного материала после каждого нагрева капсулы измерялся его гамма-спектр. Также после каждого нагрева измерялся вес капсулы с мишенным веществом для дополнительного контроля массы испаряемого мишенного вещества. Разделение мишенного вещества и целевого радионуклида стронция-82 с эффективностью, близкой к 100\%, было получено при использовании в качестве мишенного материала порошка соли $\mathrm{RbCl}[8]$. Эффективность разделения стронция и металлического рубидия в качестве мишенного вещества составила величину около 90\% [6]. На рис. 2 приведена часть гамма-спектра облученного образца хлористого рубидия естественного содержания, измеренного с помощью гамма-детектора из сверхчистого германия. Гамма-линии $550 \mathrm{keV}$ принадлежит распаду рубидия-83. По ее уменьшению в течение предварительного нагрева определялась степень испарения мишенного вещества. По гамма-линии энергии $776 \mathrm{keV}$ определялась степень испарения целевого радионуклида - стронция.

Как можно видеть из рис. 3 , если капсула с мишенным веществом $\mathrm{RbCl}$ нагревается в течение часа при температуре около $900^{\circ} \mathrm{C}$, мишенный материал полностью испаряется практически со стопроцентным (в пределах погрешности измерения 15\%) сохранением стронция в мишенной капсуле. Факт полного испарения мишенного вещества также подтверждался взвешиванием капсулы с мишенным веществом до и после нагрева при $900^{\circ} \mathrm{C}$.
Оставшийся в мишенной капсуле стронций может быть испарен из мишенной капсулы при более высокой температуре или смыт с ее внутренней поверхности небольшим количеством раствора соляной или азотной кислоты.

\section{4. Эксперименты по выделению ${ }^{67} \mathrm{Cu}$ и других радионуклидов из облученных мишенных материалов}

В экспериментах по получению ${ }^{67} \mathrm{Cu}$ в качестве мишенного материала использовался металлический цинк естественного содержания. Радионуклид ${ }^{67} \mathrm{Cu}$ с периодом полураспада $2.57 \mathrm{~d}$ считается очень перспективным радионуклидом для терапии некоторых видов злокачественных опухолей. Для разделения ${ }^{67} \mathrm{Cu}$ и мишенного материала в виде металлического цинка был использован тот же „сухой“, высокотемпературный метод, использованный для выделения изотопов стронция, основой которого является испарение мишенного материала при сохранении в мишенной капсуле целевого радионуклида. Поэтому процесс получения и выделения ${ }^{67} \mathrm{Cu}$ [9] проходил те же стадии, что и описанный выше процесс получения радионуклида ${ }^{82} \mathrm{Sr}$. На рис. 4 приведена часть гамма-спектра облученного образца металлического цинка, измеренного с помощью гамма-детектора из сверхчистого германия. Гамма-линия $1115 \mathrm{keV}$ относится к распаду ${ }^{65} \mathrm{Zn}$ с периодом полураспада $244.3 \mathrm{~d}$. Ее исчезновение в процессе нагрева является индикатором испарения мишенного вещества. Факт испарения мишенного вещества также контролировался взвешиванием капсулы с мишенным веществом до и после ее нагрева. Гамма-линия энер-

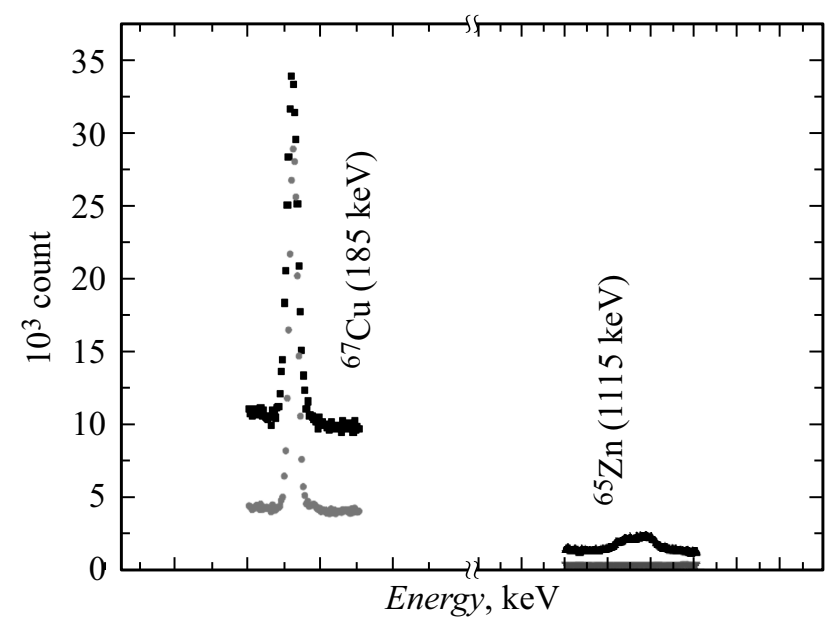

Рис. 4. Нижний спектр - атомы меди, высаженные на охлаждаемый проточной водой коллектор, испаренные из мишенной капсулы при температуре $1460^{\circ} \mathrm{C}$ в течение $2 \mathrm{~h}$ после того, как мишенное вещество - металлический цинк было полностью испарено в балластный объем при температуре $700^{\circ} \mathrm{C}$. Для сравнения показан спектр облученного мишенного вещества до нагрева (верхний спектр). 
гии $185 \mathrm{keV}$ относится к распаду целевого радионуклида ${ }^{67} \mathrm{Cu}$ с периодом полураспада $2.57 \mathrm{~d}$. Уменьшение этой линии в процессе нагрева показывает степень испарения ${ }^{67} \mathrm{Cu}$. На рисунке показана часть спектра капсулы с облученным мишенным веществом (верхний спектр) и спектр испаренных атомов меди, высаженных на охлаждаемый коллектор при температуре $1460^{\circ} \mathrm{C}$ после того, как мишенное вещество - металлический цинк - было полностью испарено в балластный объем при температуре $700^{\circ} \mathrm{C}$. Как можно видеть из приведенного рисунка, в результате двух последовательных нагревов капсулы с мишенным веществом произошло полное разделение мишенного вещества и целевого радионуклида ${ }^{67} \mathrm{Cu}$. Данный факт также был подтвержден взвешиванием капсулы с мишенным веществом до и после первого нагрева. В приведенном спектре облученного мишенного вещества также видна гаммалиния энергии $1120 \mathrm{keV}$, относящаяся к распаду ${ }^{46} \mathrm{Sc}$ $\left(T_{1 / 2}=83.8 \mathrm{~d}\right)$. Она отсутствует на коллекторе так, как скандий, будучи труднолетучим элементом, полностью остается в мишенном контейнере даже после его нагрева при температуре, значительно превышающей температуру полного испарения целевого радионуклида ${ }^{67} \mathrm{Cu}$. В первых проведенных экспериментах по разделению мишенного вещества металлического цинка и радиоактивных атомов меди была получена величина эффективности отделения мишенного вещества лучше 99\%. При этом эффективность извлечения и сбора целевого радионуклида ${ }^{67} \mathrm{Cu}$ оказалась равной $(90 \pm 15) \%$.

Как показали проведенные эксперименты, для того чтобы использовать высокотемпературный метод разделения мишенного вещества и целевого радионуклида, совсем не обязательно знать величины энергий адсорбции их атомов на внутренней поверхности мишенного контейнера. Для прогнозирования эффективного разделения достаточно хорошим ориентиром является различие в давлении насыщенных паров продукта реакции и мишенного вещества, или различие в температурах их кипения. Этот факт хорошо демонстрируется вышепредставленными результатами по выделению стронция и меди из облученных мишенных веществ. Для рубидия и стронция температуры кипения равны соответственно 688 и $1382^{\circ} \mathrm{C}$, а для цинка и меди 907 и $2562^{\circ} \mathrm{C}$. Здесь необходимо указать, что разработанный высокотемпературный метод выделения целевых радионуклидов путем предварительного испарения мишенного вещества успешно может быть использован только для выделения радионуклидов с периодом полураспада большим, чем время полного испарения мишенного вещества. В обратном случае могут быть значительные потери целевого радионуклида из-за его распада в процессе высокотемпературного выделения. Для подтверждения факта зависимости скорости выделения различных продуктов реакций из облученного мишенного вещества от их температуры кипения были проведены эксперименты по высокотемпературному выделению в вакууме радиоизотопов различных элементов из мишенного вещества в виде металлической меди [9]. Схема эксперимента

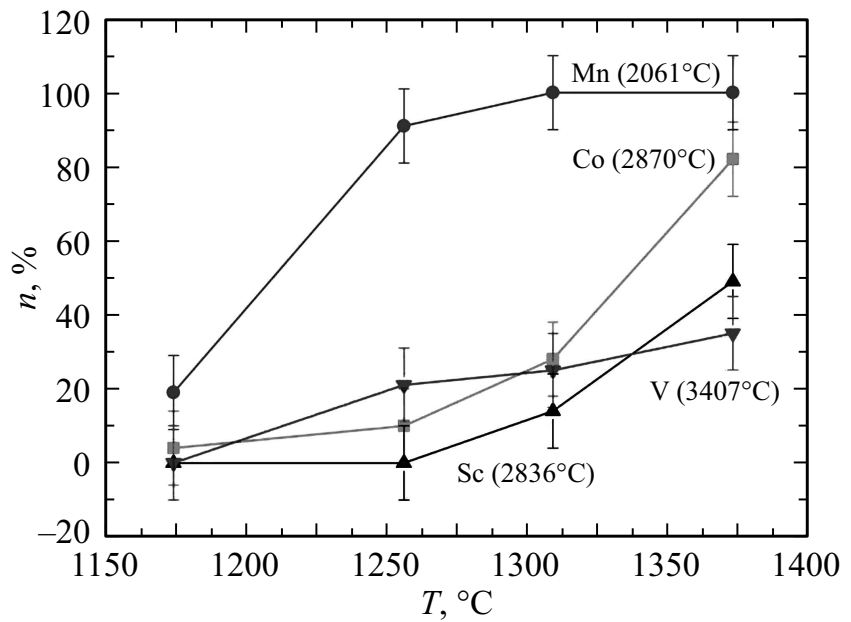

Рис. 5. Испаренные фракции радионуклидов при различных температурах, образованных в мишени из металлической меди при облучении протонами $100 \mathrm{MeV}$. В правой части рисунка в скобках даны температуры кипения указанных элементов.

была полностью идентична обсужденным выше. Облученный в пучке протонов с энергией $1000 \mathrm{MeV}$ образец металлической меди естественного содержания, помещенный в танталовую капсулу, медленно нагревался в вакууме до температуры $1500^{\circ} \mathrm{C}$. Испарение вещества мишени контролировалось взвешиванием после каждого нагрева образца. Испарение образовавшихся в процессе облучения долгоживущих радионуклидов измерялось по уменьшению площадей их гамма-линий после каждого акта нагрева. На рис. 5 показаны испаренные фракции радиоизотопов различных элементов, образованных в меди при облучении протонами $1000 \mathrm{MeV}$.

Величина испаренной фракции определялась из следующего соотношения:

$$
n=\left[\left(A_{1}-A_{2}\right) / A_{1}\right] \cdot 100 \%,
$$

где $A_{1}$ - площадь гамма-линии данного радионуклида до процесса испарения при определенной температуре, $A_{2}$ - площадь гамма-линии данного радионуклида после процесса испарения при той же температуре.

Как видно из рис. 5, в измеренном температурном интервале испаренные фракции произведенных в реакциях на протонном пучке радионуклидов находятся в соответствии с температурами их кипения. Самая большая скорость испарения у марганца, имеющего самую низкую температуру кипения из исследуемых радионуклидов, самая медленная скорость испарения у ванадия, обладающего самой высокой температурой кипения. Отклонение от этого правила в области низких температур может быть объяснено высокой погрешностью измерений при низких температурах для труднолетучих элементов - ванадия, скандия и кобальта, когда величины испаренных фракций составляют 10-20\%. 


\section{5. Выделение ${ }^{82} \mathrm{Sr}$ и других радионуклидов из труднолетучих мишенных веществ с высокой температурой кипения}

Перспективным направлением в ядерной медицине является изучение возможности использования для диагностики радионуклидов с более короткими временами жизни, чем у изотопов, применяемых в настоящее время. Это позволило бы значительно уменьшить дозу радиоактивного облучения, получаемую пациентом.

Также важной задачей остается получение радиоактивных источников высокой чистоты, что требует высокоэффективного выделения нужного радионуклида из смеси изотопов, произведенных в мишени. Обе эти задачи могут быть успешно решены использованием в режиме „в линию“ электромагнитных масс-сепараторов, позволяющих получать изотопически чистые пучки целевых радионуклидов.

Принцип действия подобной системы, называемой ISOL (Isotope Separator On-Line) установкой, состоит в следующем. Пучок протонов (или других частиц нейтронов, легких или тяжелых ионов) бомбардирует мишенное устройство, находящееся в вакуумной камере и нагретое до температуры $1800-2200^{\circ} \mathrm{C}$. Продукты реакций, диффундируют в материале мишени, термализуются, становятся нейтральными атомами и попадают в ионный источник, где происходит их ионизация. Полученные ионы ускоряются электрическим полем вытягивающего электрода и формируются в ионный пучок с помощью системы электростатических линз. Моноэнергетический пучок ионов поступает в анализирующий магнит масс-сепаратора, где под действием магнитного поля происходит разделение пучков ионов с различными массовыми числами. Далее разделенные пучки ионов поступают в камеру разводки ионных пучков, откуда и доставляются к ионным коллекторам для имплантации. Масс-сепараторный метод обеспечивает преимущества получения источников радиоизотопов высокой чистоты, причем несколько разделенных по массам нуклидов могут накапливаться одновременно. Недостатком масссепараторного метода является зависимость выхода получаемых радиоизотопов от эффективности ионизации атомов в ионном источнике: т. е. при расчетах ожидаемой активности необходимо принимать во внимание значение данной характеристики. Например, для Rb значение эффективности ионизации $\varepsilon_{i}$ составляет около $80 \%$; для $\mathrm{Sr}$, In, Ra: $\varepsilon_{i} \sim 20-40 \%$; для I (получение отрицательных ионов): $\varepsilon_{i} \sim 20-30 \%$ [10].

\section{6. Получение ${ }^{82} \mathrm{Sr}$ из мишени на основе карбида иттрия (мишень для масс-сепараторного метода производства стронция)}

Масс-сепараторный метод позволяет исключить из получаемых источников генераторного радионуклида ${ }^{82} \mathrm{Sr}$ почти в три раза более долгоживущий ${ }^{85} \mathrm{Sr}$, который всегда там присутствует при использовании традиционных методов выделения. Тем самым исключается возможность его неконтролируемого проникновения в кровеносную систему пациента, что иногда случается при использовании $\mathrm{Sr} / \mathrm{Rb}$-генераторов. Карбид иттрия высокой плотности в виде таблеток был специально изготовлен для первых экспериментов для последующего его возможного использования в качестве мишенного вещества в масс-сепараторном методе получения ${ }^{82} \mathrm{Sr}$. Облученный на пучке синхроциклотрона ПИЯФ карбид иттрия помещался в $\mathrm{Ta}-\mathrm{W}$-контейнер, изготовленный как прототип мишенного устройства, по своим характеристикам идентичный мишенному устройству [6], которое будет использовано для получения ${ }^{82} \mathrm{Sr}$ на РИЦ-80. Мишенный контейнер помещался в вакуумный стенд и нагревался до высокой температуры для извлечения полученных нуклидов из мишенного вещества, которое в силу его термостойкости и низкого давления насыщенных паров при данной (рабочей) температуре полностью сохраняется в мишенном контейнере. Этот принцип используется для извлечения из мишени продуктов ядерных реакций в системах ISOL, использующих масссепараторы, работающие в линию на пучках заряженных частиц для проведения исследований по ядерной физике. Таблетки из $\mathrm{YC}_{2}$ (дикарбида иттрия), облученные на пучке протонов с энергией $1 \mathrm{GeV}$ синхроциклотрона ПИЯФ, помещали в графитовый цилиндр, вставленный в танталовый контейнер. Центральная часть контейнера соединялась с вольфрамовой трубкой с отверстием $2 \mathrm{~mm}$ в диаметре. Радионуклиды, содержащиеся в мишенном материале, диффундировали в процессе нагревания до $2000-2100^{\circ} \mathrm{C}$ из мишенного вещества дикарбида иттрия к вольфрамовой трубке (также нагретой до $2000^{\circ} \mathrm{C}$ ), проходили через нее и оседали на коллекторе из танталовой фольги, установленном на охлаждаемом медном фланце. Специально собранный вакуумный стенд для тестирования высокотемпературных мишенных устройств позволяет рассеивать мощность до $9 \mathrm{~kW}$, что соответствует мощности, вносимой в мишень пучком протонов ускорителя Ц-80.

Исследуемый прототип мишенного устройства позволяет получать и выделять радионуклиды из таких материалов (используемых в качестве мишенных веществ), как тугоплавкие металлы и их карбиды, жидкие металлы и композиты. Данный прототип можно использовать как в качестве мишенного устройства для масссепараторной станции, так и как мишень для метода высокотемпературного „сухого“ выделения радионуклидов (без использования масс-сепаратора). В разд. 6 настоящей работы представлены результаты исследований мишенных материалов, которые будут использоваться для масс-сепараторного метода получения радионуклидов. Поскольку выделение стронция из дикарбида иттрия характеризуется сравнительно медленными диффузионноэффузионными процессами, на первом этапе мишень нагревали до $1500-1800^{\circ} \mathrm{C}$, чтобы выделить продукты 

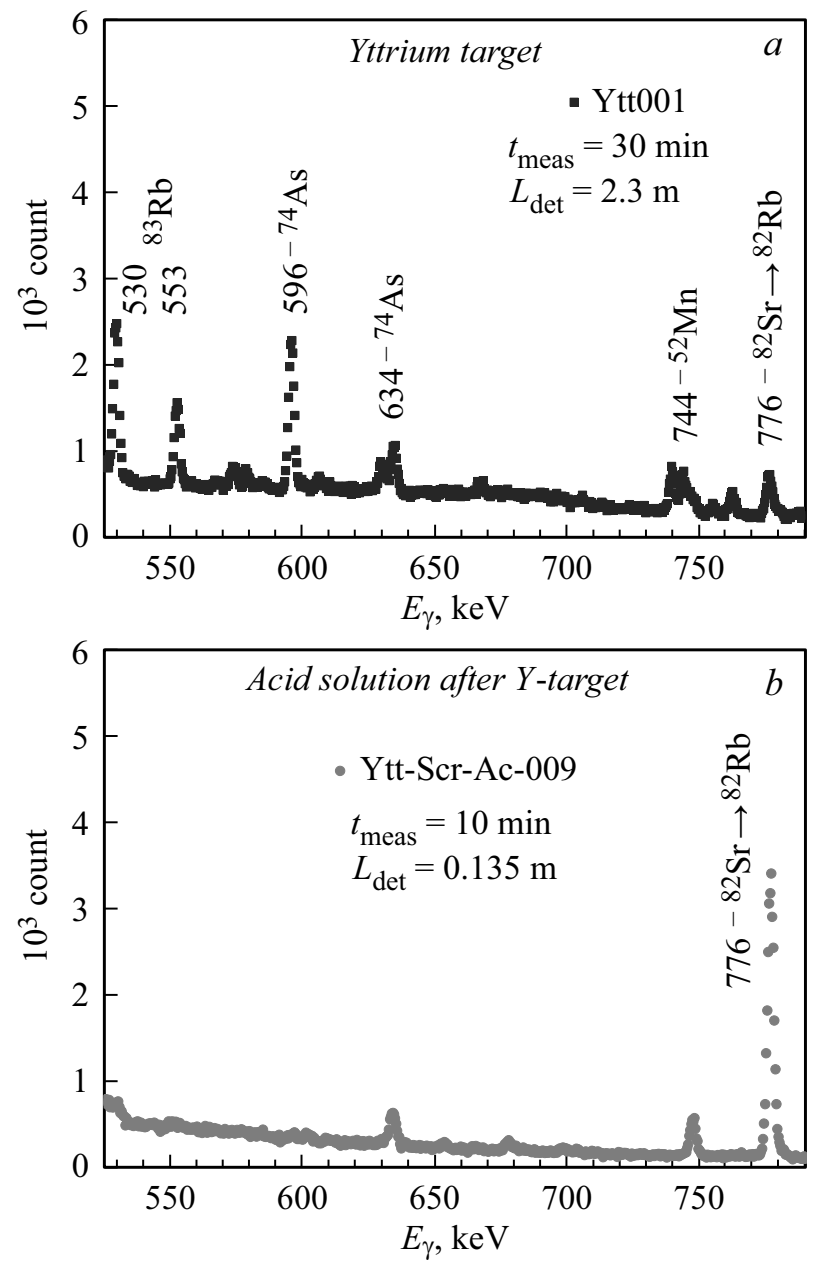

Рис. 6. $a-\gamma$-спектр облученного образца дикарбида иттрия до его нагревания на вакуумном стенде; $b-\gamma$-спектр радиоактивного источника, выделенного на охлаждаемый коллектор в результате второго нагрева мишени при температуре $1950-2000^{\circ} \mathrm{C}$. Гамма-линия энергии $776 \mathrm{keV}$ из распада изотопа ${ }^{82} \mathrm{Rb}$, используемого для ПЭТ диагностики. На рисунке указаны величины: $t_{\text {meas }}-$ время измерения и $L_{\mathrm{det}}-$ расстояние до детектора.

с более быстрыми диффузионно-эффузионными характеристиками (например, изотопы $\mathrm{Rb}$ и $\mathrm{Mn}$ ). На следующем этапе при более высокой температуре из мишени селективно выделяли стронций. На рис. 6, $a$ представлен $\gamma$-спектр облученного образца карбида иттрия до нагревания на вакуумном стенде. На рис. $6, b$ показан $\gamma$-спектр радиоактивного источника, выделенного на охлаждаемый коллектор в результате нагрева мишени до высокой температуры. Из сравнения приведенных спектров можно видеть, что на этапе нагрева мишени до $1500-1800^{\circ} \mathrm{C}$ из нее удалось устранить почти все радиоактивные продукты, кроме изотопа стронция. Окончательная эффективность выделения стронция на охлаждаемый коллектор при нагревании мишени до $1950-2000^{\circ} \mathrm{C}$ в течение $10 \mathrm{~h}$ составила около $90 \%$. Таким образом, мишень на основе карбида иттрия - это хороший кандидат для получения изотопа ${ }^{82} \mathrm{Sr}$ масс-сепараторным методом. Данный материал обладает подходящими характеристиками (температура плавления, температура кипения) для высокотемпературного мишенного устройства, соединенного с источником поверхностной ионизации.

\section{7. Получение разделенных по массам изотопов ${ }^{223,224} \mathrm{Ra}$ и ${ }^{225} \mathrm{Ac}$ в режиме "on-line" c масc-сепаратором из мишени карбида урана высокой плотности}

Изотопы Ra-223,224 и Аc-225, распадающиеся альфараспадом, получались разделенными на коллекторе масс-сепаратора ИРИС (Исследование Радиоактивных Изотопов на Синхроциклотроне) [11], работающем в режиме „on-line“ с синхроциклотроном ПИЯФ СЦ-1000. Для накопления радионуклидов радия и актиния на подвижный коллектор лентопротяжного устройства проводилась стандартная процедура, используемая при проведении экспериментов на установке ИРИС: путем пошагового изменения магнитного поля магнита масс-сепаратора на коллектор (ленту лентопротяжного устройства) поочередно выводились ионные пучки с массовыми числами 223, 224, 225 и после их имплантациинакопления и перемещения к соответствующим детекторам измерялись гамма-, а также альфа-спектры имплантированных источников. Для определения дисперсии в области масс 220-230 проводилась одновременная высадка нескольких изотопных пучков в данной массовой области на коллектор масс-сепаратора, расположенный в фокальной плоскости его магнита. На рис. 7 показаны разделенные по массам изотопы, имплантированные в алюминиевую фольгу, расположенную в фокальной плоскости магнита масс-сепаратора в массовой области

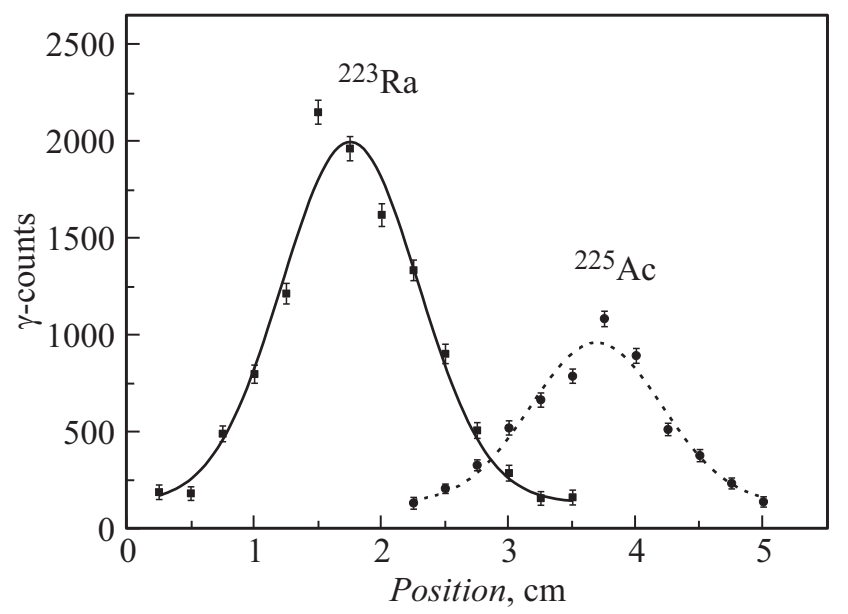

Рис. 7. Разделенные по массам изотопы в массовой области 223-225 (радий-223 и актиний-225) в фокальной плоскости магнита масс-сепаратора, идентифицированные по их соответствующим гамма-линиям. 
223-225 (изотопы радия и актиния), измеренные по их гамма-линиям.

Как можно видеть из рис. 7, массовые линии 223, 225 разрешаются, перекрываясь своими хвостами. Разрешение может быть значительно улучшено путем более тщательной юстировки ионного источника, уменьшением его выходного отверстия, а также использованием щелей, установленных перед фокальной плоскостью магнита, позволяющих обрезать „хвосты“ массовых линий. На рис. 8 показаны альфа-спектры радионуклидов ${ }^{223} \mathrm{Ra}$, ${ }^{225} \mathrm{Ac}$, разделенных с помощью масс-сепаратора и имплантированных в алюминиевую фольгу-коллектор, расположенную в фокальной плоскости масс-сепаратора. Как уже говорилось выше, для определения дисперсии в области атомных масс 220-230 проводилась одновременная высадка нескольких изотопных пучков в данной массовой области на коллектор масс-сепаратора, расположенный в фокальной плоскости его магнита. Среди приведенных спектров отсутствует спектр ${ }^{224} \mathrm{Ra}$ $\left(T_{1 / 2}=3.66 \mathrm{~d}\right)$, так как измерения имплантированных источников проводились через три недели после их накопления. Однако масс-сепарированный ${ }^{224} \mathrm{Ra}$ может

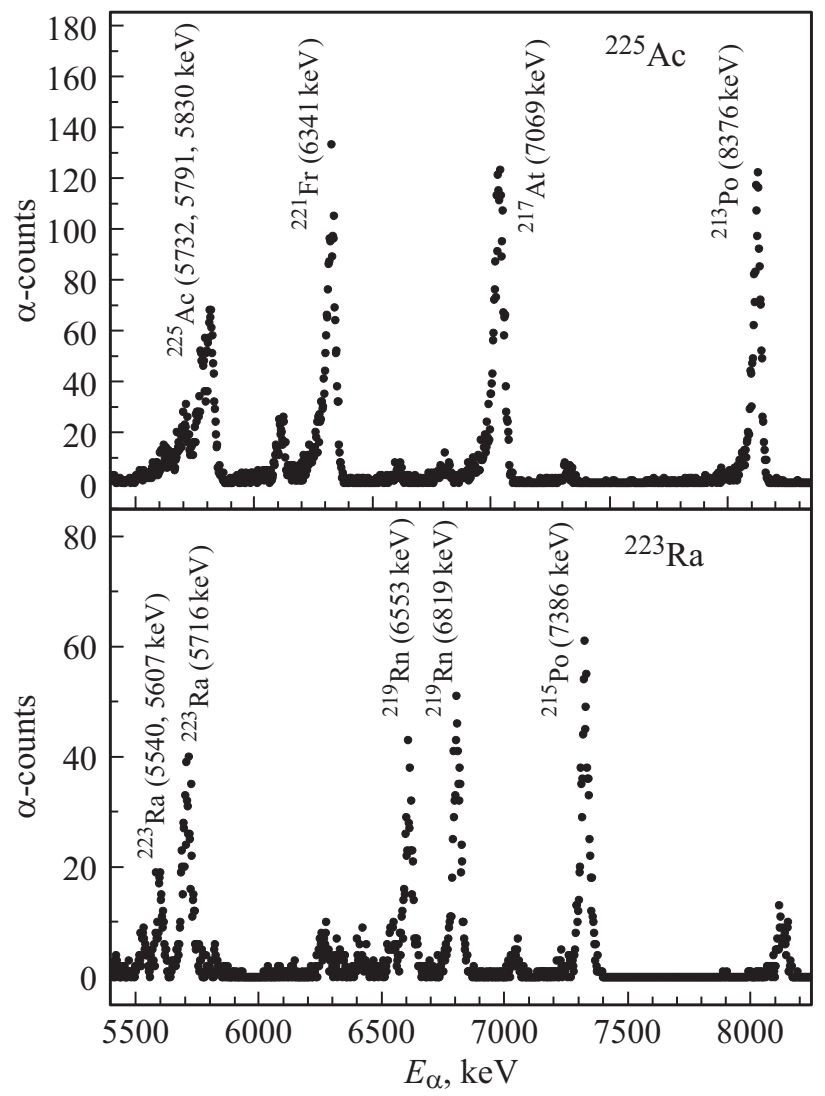

Рис. 8. Альфа-спектры разделенных с помощью масссепаратора изотопов ${ }^{225} \mathrm{Ac} \quad$ и $\quad{ }^{223} \mathrm{Ra} \quad\left({ }^{225} \mathrm{Ac} \quad\left(T_{1 / 2}=10 \mathrm{~d}\right)\right.$ $\rightarrow{ }^{221} \mathrm{Fr} \quad\left(T_{1 / 2}=4.9 \mathrm{~min}\right) \quad \rightarrow \quad{ }^{217} \mathrm{At} \quad\left(T_{1 / 2}=32.3 \mathrm{~ms}\right) \quad \rightarrow$ ${ }^{217} \mathrm{Bi} \quad\left(T_{1 / 2}=45.51 \mathrm{~min}\right) \rightarrow{ }^{213} \mathrm{Po} \quad\left(T_{1 / 2}=4.2 \mu \mathrm{s}\right) \rightarrow{ }^{209} \mathrm{~Pb}$ $\left.\left(T_{1 / 2}=2.25 \mathrm{~h}\right)\right)$ и $\left({ }^{223} \mathrm{Ra}\left(T_{1 / 2}=11.4 \mathrm{~d}\right) \rightarrow{ }^{219} \mathrm{Rn}\left(T_{1 / 2}=3.96 \mathrm{~s}\right)\right.$ $\rightarrow{ }^{215} \mathrm{Po}\left(T_{1 / 2}=1.8 \mathrm{~ms}\right) \rightarrow{ }^{211} \mathrm{~Pb}\left(T_{1 / 2}=36.1 \mathrm{~min}\right) \rightarrow{ }^{211} \mathrm{Bi}$ $\left.\left(T_{1 / 2}=2.14 \mathrm{~min}\right) \rightarrow{ }^{207} \mathrm{Tl}\left(T_{1 / 2}=4.77 \mathrm{~min}\right)\right)$, имплантированных в алюминиевую фольгу-коллектор.
Эффективность ионизации целевых радионуклидов при их получении на масс-сепараторе

\begin{tabular}{c|c|c|c}
\hline Элемент & $\begin{array}{c}\text { Потенциал } \\
\text { ионизации, } \\
\mathrm{eV}\end{array}$ & $\begin{array}{c}\text { Эффективность, } \\
\%\end{array}$ & $\begin{array}{c}\text { Погрешность } \\
\text { определения } \\
\text { эффективности, \% }\end{array}$ \\
\hline $\mathrm{Cs}$ & 3.9 & 51 & 15 \\
$\mathrm{Rb}$ & 4.2 & 47 & 10 \\
$\mathrm{Ra}$ & 5.3 & 38 & 10 \\
$\mathrm{In}$ & 5.8 & 33 & 8 \\
$\mathrm{Tl}$ & 6.1 & 21 & 8
\end{tabular}

быть получен из той же облученной мишени, где он образуется в результате $\alpha$-распада его материнского ядpa ${ }^{228} \mathrm{Th}\left(T_{1 / 2}=1.9 \mathrm{y}\right)$, с использованием ее повторного нагрева до рабочей температуры $2200-2400^{\circ} \mathrm{C}$.

Как можно видеть по соответствующим альфа-линиям на рис. 8 , радионуклиды ${ }^{223} \mathrm{Ra}$ и ${ }^{225} \mathrm{Ac}$, накопленные на коллекторе в фокальной плоскости масс-сепаратора, достаточно хорошо разделяются. Однако на обоих спектрах видна перекрестная примесь данных радионуклидов в виде слабых альфа-линий - ${ }^{223} \mathrm{Ra}$ в спектре ${ }^{225} \mathrm{Ac}$ и наоборот ${ }^{225} \mathrm{Ac}$ в спектре ${ }^{223} \mathrm{Ra}$. Возможность и методы устранения „хвостов““ соседних линий в данной области масс были обсуждены выше. Для получаемых радионуклидов по их измеренным выходам были определены эффективности ионизации, представленные в таблице. Необходимо отметить, что измеренные эффективности ионизации, как это видно из приведенной таблицы, имеют высокие значения, что было обеспечено использованием в качестве ионного источника вольфрамовой трубки с повышенной работой выхода $(5 \mathrm{eV})$ внутренней поверхности.

\section{Заключение}

Полученные в настоящей работе результаты будут использованы на изотопном комплексе РИЦ-80, где, наряду с указанными, планируется производство других радиоизотопов. Проведенные исследования продемонстрировали высокую эффективность использования в качестве мишенного вещества монокарбида урана высокой плотности. Для выделения целевых радионуклидов из облученного мишенного вещества использовался как метод высокотемпературного выделения в высоком вакууме из облученного мишенного вещества, так и метод с использованием масс-сепаратора. Было показано, что оба метода обеспечивают высокую эффективность выделения произведенных радиоизотопов из мишенного вещества при его рабочей температуре $2300-2400^{\circ} \mathrm{C}$. Также было показано, что эффективность ионизации радия в источнике поверхностной ионизации из монокристалла вольфрама с работой выхода внутренней поверхности $5 \mathrm{eV}$ составляет для указанных радионуклидов около $80 \%$ и может быть повышена до $100 \%$. 
Это говорит о том, что масс-сепараторный метод, обеспечивая одновременное получение сразу трех вышеуказанных радиоизотопов альфа-распадчиков, практически не будет вносить потерь в активности получаемых радионуклидов. В заключение необходимо указать, что мишенное вещество карбид тория, планируемое для получения вышеуказанных радионуклидов на РИЦ-80, обладает сходными с монокарбидом урана физикохимическими свойствами - температурой плавления, температурой кипения, плотностью. Его использование в качестве мишенного вещества обеспечит значительное (больше, чем на порядок) увеличение выходов целевых радионуклидов.

\section{Список литературы}

[1] Artamonov S.A., Ivanov E.M., Mikheev G.F., Mironov Yu.T., Riabov G.A., Mudrolubov V.G. High Energy Physics Division: Main scientific Activities / Ed. by G.D. Alkhazov. Gatchina. 2013. P. 332-338.

(http://hepd.pnpi.spb.ru/hepd/articles/PNPI_2007-2012.pdf).

[2] Panteleev V.N., Barzakh A.E., Batist L.Kh., Fedorov D.V., Filatova A.M., Ivanov V.S., Mezilev K.A., Moroz F.V., Molkanov P.L., Orlov S.Yu., Volkov Yu.M. High Energy Physics Division: Main scientific Activities / Ed. by G.D. Alkhazov Gatchina. 2013. P. 278-282. (http://hepd.pnpi.spb.ru/hepd/articles/PNPI_2007-2012.pdf).

[3] Panteleev V.N., Barzakh A.E., Batist L.Kh., Fedorov D.V., Filatova A.M., Ivanov V.S., Moroz F.V., Molkanov P.L., Orlov S.Yu., Volkov Yu.M. Proceedings of the Third International Conference on Radiation and Applications in Various Fields of Research / Ed. by G. Ristić. Montenegro: Budva, 2015. P. 51-56.

[4] Panteleev V.N., Barzakh A.E., Batist L.Kh., Fedorov D.V., Ivanov V.S., Molkanov P.L., Moroz F.V., Orlov S.Yu., Volkov Yu.M. // Rev. Sci. Instrum. 2015. Vol. 86. P. 123510.

[5] Пантелеев В.Н., Барзах А.Е., Батист Л.Х., Федоров Д.В., Иванов В.С., Кротов С.А., Мороз Ф.В., Молканов П.Л., Орлов С.Ю., Волков Ю.М. // Медицинская физика. 2016. № 2. C. $47-55$.

[6] Panteleev V.N., Barzakh A.E., Fedorov D.V., Filatova A.M., Ivanov V.S., Mezilev K.A., Moroz F.V., Molkanov P.L., Orlov S.Yu. High Energy Physics Division: Main scientific Activities / Ed. by G.D. Alkhazov. Gatchina. 2013. P. 347354.

(http://hepd.pnpi.spb.ru/hepd/articles/PNPI_2007-2012.pdf).

[7] Kirchner R. // Nucl. Instr. Meth. Phys. Res. B 26. 1987. P. 204-212.

[8] Пат. РФ № 2598089. Способ получения радионуклида стронция-82 / В.Н. Пантелеев. 2016.

[9] Panteleev V.N., Barzakh A.E., Batist L.Kh., Fedorov D.V., Ivanov V.S., Krotov S.A., Moroz F.V., Molkanov P.L., Orlov S.Yu., Volkov Yu.M. // RAD Conf. Proceed. 2017. Vol. 2. P. 43-47. DOI: $10.21175 /$ RadProc. 2017.10

[10] Kirchner R. // Nucl. Instr. Meth. 1990. A 292. P. 203-208.

[11] Panteleev V.N., Barzakh A.E., Fedorov D.V., Moroz F.V., Orlov S.Yu., Seliverstov M.D., Volkov Yu.M., Tecchio L., Andrighetto A. // Rev. Sci. Instrum. 2002. Vol. 73. N 2. P. 738-740. 